\title{
Test yourself: Recurrent right groin lump
}

\author{
Henry Chi Kit Kwok ${ }^{1}$ (1) $\cdot$ Mary Susan Brady ${ }^{2}$ Narasimhan P. Agaram ${ }^{3} \cdot$ Sinchun Hwang ${ }^{1}$
}

Received: 13 October 2021 / Revised: 17 November 2021 / Accepted: 19 November 2021 / Published online: 26 November 2021 (c) ISS 2021

\section{Answer}

Femoral hernia

\section{Discussion}

The right groin sonography (Fig. 1) demonstrates an anechoic lobulated mass with posterior acoustic enhancement consistent with a cystic nature. There is no internal solid component or vascularity. The mass is located between the pubic bone medially and the external iliac vessels laterally. Magnetic resonance imaging (MRI) demonstrates a uniformly $\mathrm{T} 1$ hypointense and $\mathrm{T} 2$ hyperintense mass without contrast enhancement (Fig. 2). The mass was excised, but 4 years later, an MRI examination shows a recurrent mass of a similar cystic appearance. On both sonography and MRI, the mass shows a tapering end that extends posteriorly and superiorly in the groin, and this appearance mimics a "speech box" (Fig. 3). This imaging feature and the location of the mass confirm the diagnosis of femoral hernia, which was subsequently repaired. The histology of the hernia sac consists of a monolayer of mesothelial cells lining fibroadipose tissue (Fig. 4).

A femoral hernia is a protrusion of the peritoneal sac through the femoral ring into the femoral canal. Femoral

The case presentation can be found at: https://doi.org/10.1007/ s00256-021-03963-w.

Henry Chi Kit Kwok

kwokh@mskcc.org

1 Department of Radiology, Memorial Sloan Kettering Cancer Center, 1275 York Ave, New York, NY 10065, USA

2 Department of Surgery, Memorial Sloan Kettering Cancer Center, New York, NY, USA

3 Department of Pathology, Memorial Sloan Kettering Cancer Center, New York, NY, USA hernias have a much lower incidence than inguinal hernias but carry a higher complication rate [1]. Clinical diagnosis is often difficult especially in early cases, with up to $40 \%$ missed at presentation [2]. Imaging, therefore, plays an essential role in management, as an early and accurate diagnosis of femoral hernia can help prevent potential complications of delayed diagnosis such as bowel strangulation and avoid unnecessary investigation or inadequate surgical treatment.

While the diagnosis of larger femoral hernias containing bowel or peritoneal fat is relatively straightforward, early femoral hernias may contain only peritoneal fluid and can be difficult to distinguish from other cystic lesions on imaging. As a case in point, we have failed to diagnose such femoral hernia in the present patient on a number of occasions, resulting in the need for a second surgical procedure. In hindsight, "availability bias" [3] might have resulted in the inability to consider femoral hernia as a differential diagnosis due to its lower probability relative to other conditions in the patients we serve, while "alliterative error" [3] might have perpetuated the error subsequently. Moreover, Valsalva maneuver, a technique which might potentially alert the radiologists to the diagnosis [4], was not performed during sonography.

Thus, femoral hernia should be considered in the differential diagnoses whenever a cystic groin mass is encountered at or near the femoral ring or canal, inferior to the inguinal ligament between the pubic tubercle and the femoral vein. Other considerations include a hydrocele of the Canal of Nuck (in women), lymphangioma, postoperative lymphocele or seroma, a cystic or necrotic lymph node and an epidermoid inclusion cyst. In these situations, a careful search for the subtle "speech box" sign [5] can lead to the correct diagnosis of femoral hernia. This sign arises when a fluid-filled structure, in this case a hernia sac, narrows rapidly towards the hernia neck at the femoral ring. This sign is not unique 


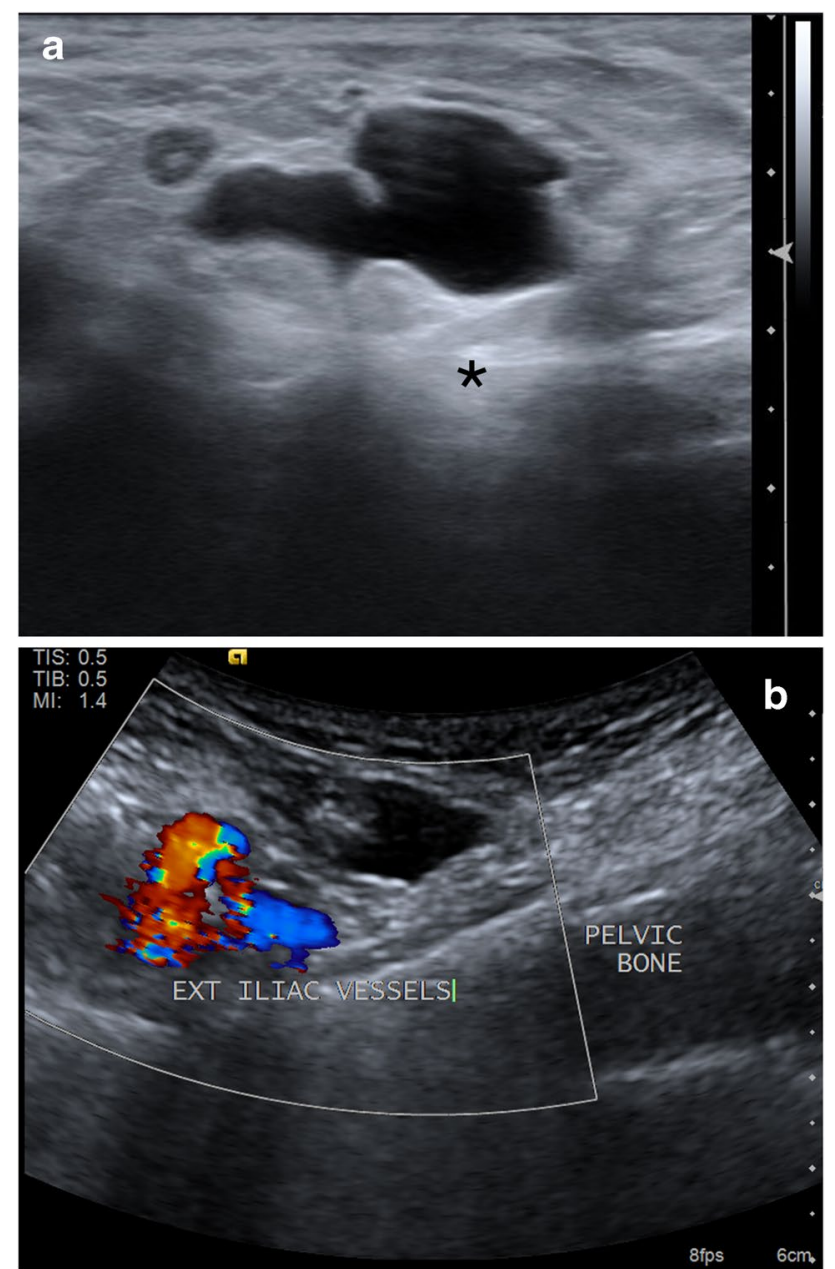

Fig. 1 Ultrasound before initial resection. (a) Transverse gray-scale and (b) transverse color Doppler sonographic images of the right groin show an anechoic mass with posterior acoustic enhancement (*) and without vascular flow

to femoral hernias, and similar findings have been described in other entities such as popliteal cysts [6], where the tapering extends towards the medial knee joint. With sonography, Valsalva maneuver may also be performed to verify the diagnosis [4].

In summary, imaging diagnosis of femoral hernia can be potentially difficult and radiologists must be cognizant of this possible differential diagnosis whenever a cystic lesion is encountered at its characteristic location. Recognizing the "speech box" sign in these situations is helpful in securing the correct diagnosis.
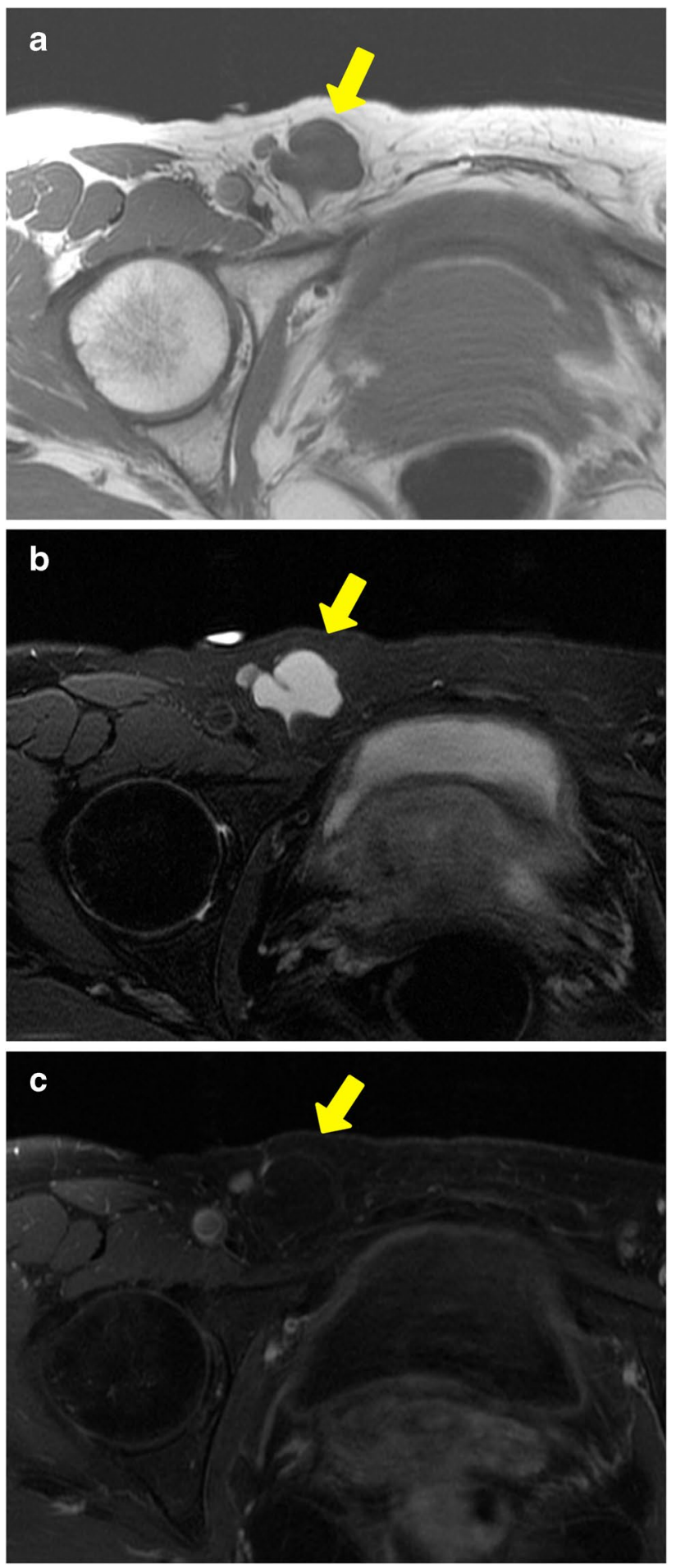

Fig. 2 MRI before initial resection. (a) Axial T1, (b) axial T2 fat-saturated, and (c) post-contrast axial T1 fat-saturated MRI images of the pelvis demonstrate a uniformly $\mathrm{T} 1$ hypointense and $\mathrm{T} 2$ hyperintense mass (arrows) with no contrast enhancement 

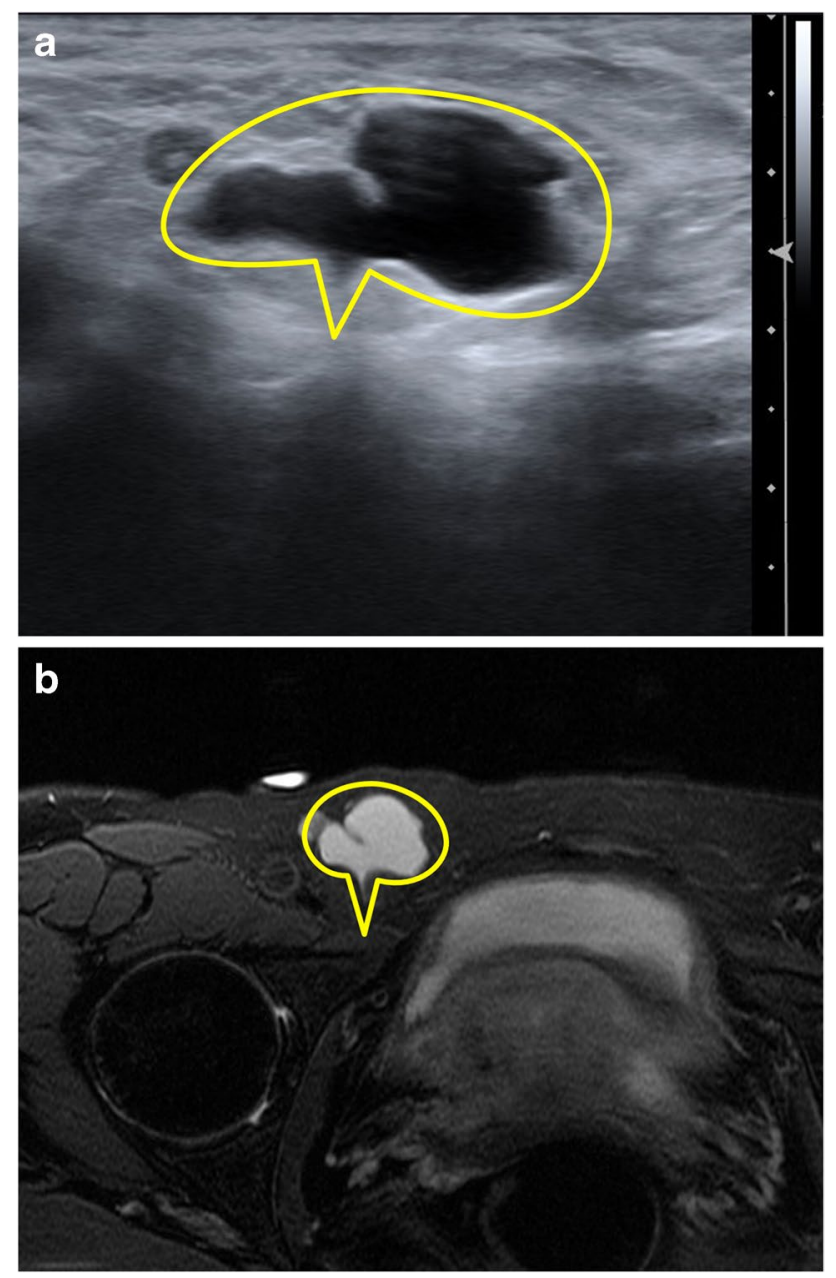

Fig. 3 Speech box sign. (a) Transverse gray-scale sonographic image and (b) axial T2 fat-saturated MRI image show a tapering end that extends posteriorly in the groin. The appearance mimics a "speech box" (yellow outline)

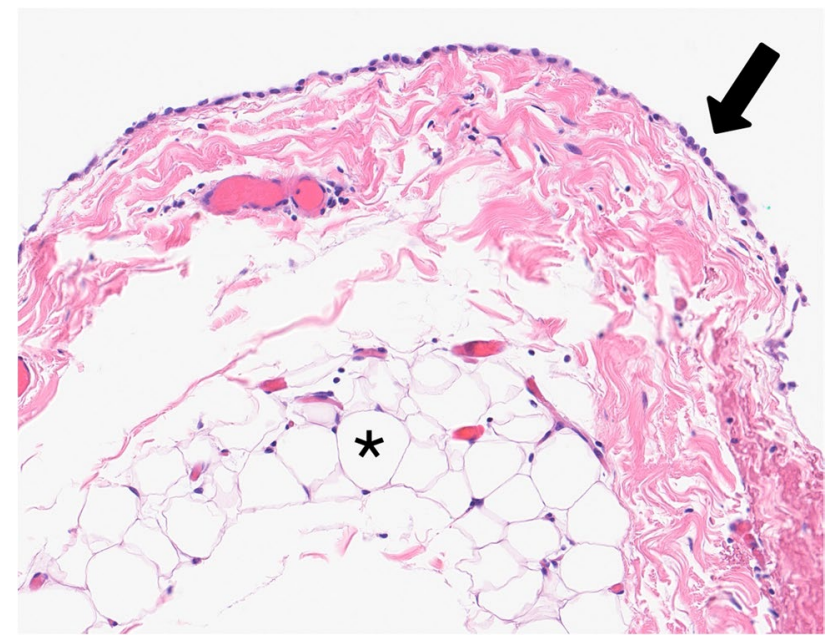

Fig. 4 Histology images show fibroadipose tissue $\left(^{*}\right)$ lined by a monolayer of mesothelial cells (arrow), forming the wall of hernia sac (H\&E stain, $400 \times$ )

\section{Declarations}

Conflict of interest The authors declare no competing interests.

Ethics approval and consent to participate Approval from the Institutional Review Board was obtained and in keeping with the policies for a non-human subject research; institutional review board oversight was not required. Consent has been obtained from the patient for the use of their data and images in this case report.

\section{References}

1. Gallegos NC, Dawson J, Jarvis M, Hobsley M. Risk of strangulation in groin hernias. Br J Surg. 2005;78(10):1171-3.

2. Nilsson $\mathrm{H}$, Nilsson E, Angerås U, Nordin P. Mortality after groin hernia surgery: delay of treatment and cause of death. Hernia. 2011;15(3):301-7.

3. Busby LP, Courtier JL, Glastonbury CM. Bias in radiology: the how and why of misses and misinterpretations. Radiographics. 2018;38(1):236-47.

4. Jacobson JA, Khoury V, Brandon CJ. Ultrasound of the groin: techniques, pathology, and pitfalls. Am J Roentgenol. 2015;205(3):513-23.

5. Thaker S, Botchu R, Gupta H. Sonographic, "speech bubble/ speech box sign" in clinically occult femoral hernias: ultrasound findings and anatomical basis. J Ultrasound. 2021;24(3):361-6.

6. Vlychou M, Teh J. Ultrasound of muscle. Curr Probl Diagn Radiol. 2008;37(5):219-30.

Publisher's note Springer Nature remains neutral with regard to jurisdictional claims in published maps and institutional affiliations. 